\title{
EXTENSION OF BOUNDED LINEAR OPERATORS
}

\author{
Prakash Muni Bajryacharya ${ }^{1}$, Keshab Raj Phulara ${ }^{2}$ \\ ${ }^{1}$ Professor, Department of Mathematics, Central Department of Mathematics, Kritipur, Kathmandu, Nepal \\ Email Address: pmbajracharya13@gmail.com \\ ${ }^{2}$ M. Phil Scholar, Central Department of Mathematics, Kritipur, Kathmandu, Nepal \\ Email Address: krphulara@gmail.com
}

\begin{abstract}
In this article the problem entitled when does every member of a class of operators $\mathrm{T}: \mathrm{E} \rightarrow \mathrm{Y}$ admit an extension operator $\hat{\mathrm{T}}$ : $\mathrm{X} \rightarrow \mathrm{Y}$ in different approaches like injective spaces, separably injective spaces, the class of compact operators and extension into $\mathrm{C}(\mathrm{K})$ spaces has been studied.
\end{abstract}

Keywords: Injective Spaces, Separable Injective Spaces, Compact Operator, Bounded Linear Operator.

\section{Introduction}

Let $\mathrm{X}$ and $\mathrm{Y}$ be Banach spaces and let $\mathrm{E}$ be a subspace of $\mathrm{X}$. An operator $\hat{\mathrm{T}}: \mathrm{X} \rightarrow \mathrm{Y}$ is said to be an extension of an operator $\mathrm{T}: \mathrm{E} \rightarrow \mathrm{Y}$ if $\hat{\mathrm{T}} \mathrm{e}=\mathrm{Te}$ for all $\mathrm{e} \in \mathrm{E}$. The general problem discussed below is the following: when does every member of a class of operators $\mathrm{T}: \mathrm{E} \rightarrow \mathrm{Y}$ admit an extension operator $\hat{\mathrm{T}}: \mathrm{X} \rightarrow \mathrm{Y}$ (of the same class)?

We will introduce here the basic definitions and describe the main extension problems. The starting point of all extension theories is the following extension theorem for linear functional.

Theorem 0.1. [Hahn-Banach Theorem [?] Let $\mathrm{X}$ be a Banach space over the real or complex field $\mathrm{F}$ and let $\mathrm{E}$ be a subspace of $\mathrm{X}$. Then every bounded linear functional $\mathrm{e}^{*}: \mathrm{E} \rightarrow \mathrm{F}$ can be extended to a linear functional $\mathrm{x}^{*}: \mathrm{X} \rightarrow \mathrm{F}$ with $\left\|\mathrm{x}^{*}\right\|=\left\|\mathrm{e}^{*}\right\|$.

Unfortunately, such perfection is rare; few extension theories which deal with more general operator extension problems can avoid compromises. To generalize the Hahn-Banach Theorem necessarily requires some restrictions: restrictions on the spaces $\mathrm{X}$ and $\mathrm{E}$, restrictions on the range space $\mathrm{Y}$, relaxation of the norm preservation condition $\|\hat{\mathrm{T}}\|=\|\mathrm{T}\|^{1}$ or restrictions on the class of operators to be extended.

We start with a discussion about conditions on the domain space $\mathrm{E}$ which ensure, in full generality, the existence of bounded extensions of operators.

\subsection{The Injective Spaces}

Given a Banach space $X$, a subspace $E$ of $X$ and $\lambda \geq 1$, we say that the pair (E, $X)$ has the $\lambda$ extension property ( $\lambda$-EP, in short) if, for every Banach space $Y$, every operator $T: E \rightarrow Y$ admits an extension $\hat{\mathrm{T}}: \mathrm{X} \rightarrow \mathrm{Y}$ with $\|\hat{\mathrm{T}}\| \leq \lambda\|\mathrm{T}\|$. The pair $(\mathrm{E}, \mathrm{X})$ is said to have the Extension Property (EP in short) if for every space $\mathrm{Y}$, every operator $\mathrm{T}: \mathrm{E} \rightarrow \mathrm{Y}$ admits an extension $\hat{\mathrm{T}}: \mathrm{X} \rightarrow \mathrm{Y}$. 
These two properties are closely connected to the following: A Banach space $E$ is called $\lambda$-injective or a $\mathbf{P} \lambda$ space, if for every space $X$ containing $E$ there is a projection $P$ of $X$ onto $E$ with $\|P\| \leq \lambda$. $E$ is called injective (or a $\mathbf{P}$ space) if, for every $\mathrm{X}$ containing $\mathrm{E}$, there is a projection of $\mathrm{X}$ onto $\mathrm{E}$. The relations between the above properties are formulated in the following.

Theorem 0.2: Let $\mathrm{E}$ be a Banach space and let $\lambda \geq 1$. The following three assertions are equivalent,

(1.1) $\mathrm{E}$ is $\lambda$-injective

(1.2) For every space $X$ containing $E,(E, X)$ has the $\lambda$-EP

(1.3) For every pair of spaces $Y \supset X$, every operator $T: X \rightarrow E$ admits an extension $\hat{\mathrm{T}}: \mathrm{Y} \rightarrow \mathrm{E}$ with $\|\hat{\mathrm{T}}\| \leq \lambda\|\mathrm{T}\|$.

This theorem shows that $\mathrm{E}$ is injective if and only if $(\mathrm{E}, \mathrm{X})$ has the $\mathrm{EP}$ for every $\mathrm{X}$ containing $\mathrm{E}$ and this property is equivalent to (1.3) with the condition $\|\hat{\mathrm{T}}\| \leq \lambda\|\mathrm{T}\|$ omitted. By using the third property (1.3) it is easily proved that every injective space is $\lambda$-injective for some $\lambda \geq 1$.

The problem of characterizing the $\mathbf{P}$ spaces is a long standing open problem in Banach space theory.

\subsection{Separably Injective Spaces}

An infinite dimensional separable Banach space is called separably injective if, for every separable space $\mathrm{X}$ containing $\mathrm{E}$, there is a projection $\mathrm{P}$ of $\mathrm{X}$ onto $\mathrm{E} .{ }^{2}$ Given $\lambda \geq 1, \mathrm{E}$ is called $\lambda$-separably injective if the above condition is satisfied with the additional restriction $\|\mathrm{P}\| \leq \lambda$.

Theorem 0.3: Let $\mathrm{E}$ be a separable Banach space and $\lambda \geq 1$. Then the following assertions are equivalent:

(1.4) $\mathrm{E}$ is $\lambda$-separably injective

(1.5) For every separable $X$ containing E, the pair (E, X) has the $\lambda$-EP.

(1.6) Let $\mathrm{Y} \supset \mathrm{X}$ be any separable spaces. Then every operator $\mathrm{T}: \mathrm{X} \rightarrow \mathrm{E}$ admits an extension $\hat{\mathrm{T}}$ $: \mathrm{Y} \rightarrow \mathrm{E}$ with $\|\hat{\mathrm{T}}\| \leq \lambda\|\mathrm{T}\|$.

The characterization problem of separably injective spaces has been completely s o lved.

\subsection{The Class of Compact Operators}

An extension theory for compact operators was developed by J. Lindenstrauss. This theory establishes relations between extension properties of compact operators with intersection properties of balls and the special structure of the domain or range space $\mathrm{X}$.

Theorem 0.4: Let $\mathrm{W}$ be a subspace of a Banach space $\mathrm{X}$ and let $\mathrm{Q}$ be an operator from a space $\mathrm{Z}$ onto $\mathrm{X}$ so that $\|\mathrm{Q}\|=1$ and $\mathrm{Q}(\operatorname{Ball}(\mathrm{Z})) \supset \delta \mathrm{Ball}(\mathrm{X})$. Let $\mathrm{Y}$ be any Banach space and suppose that every operator $\mathrm{T}: \mathrm{Q}^{-1}(\mathrm{~W}) \rightarrow \mathrm{Y}$ admits an extension $\hat{\mathrm{T}}: \mathrm{Z} \rightarrow \mathrm{Y}$ with $\|\hat{\mathrm{T}} \mathrm{II} \leq \lambda\| \mathrm{T} \|$. Then any $\mathrm{S}:$ $\mathrm{W} \rightarrow \mathrm{Y}$ admits an extension $\hat{\mathrm{S}}: \mathrm{X} \rightarrow \mathrm{Y}$ with $\|\hat{\mathrm{S}}\| \leq \lambda \delta^{-1}\|\mathrm{~S}\|$.

\subsection{Extension into $\mathrm{C}(\mathrm{K})$ Spaces}

By restricting the range space $Y$ to the class of $L_{1}(\Omega, \mu)$ preduals, we can nicely extend any compact operator $\mathrm{T}: \mathrm{E} \rightarrow \mathrm{Y}$ to a compact operator $\hat{\mathrm{T}}: \mathrm{X} \rightarrow \mathrm{Y}$ with $\|\hat{\mathrm{T}}\|=\|\mathrm{T}\|$, whenever $\mathrm{E} \subset \mathrm{X}$. Does the same restriction on $\mathrm{Y}$ make any operator $\mathrm{T}: \mathrm{E} \rightarrow \mathrm{Y}$ extendable? The answer is negative in general. 
However, positive partial results can be obtained in the special case of $\mathrm{Y}=\mathrm{C}(\mathrm{K})$, the space of continuous functions on a compact Hausdorff space $\mathrm{K}$. For example, Lindenstrauss and Pełczyński proved the following:

Theorem 0.5: ([Li-Pe 2] Theorem 3.1]). Let $\mathrm{E}$ be a subspace of $\mathrm{c}_{0}$ and let $\mathrm{Y}=\mathrm{C}(\mathrm{K})$, for some compact Hausdorff space $\mathrm{K}$. Then any operator $\mathrm{T}: \mathrm{E} \rightarrow \mathrm{Y}$ admits, for every $\varepsilon>0$, an extension $\hat{\mathrm{T}}$ : $\mathrm{c}_{0} \rightarrow \mathrm{Y}$ with $\|\hat{\mathrm{T}}\| \leq(1+\varepsilon)\|\mathrm{T}\|$.

${ }^{3} 1 \mathrm{t}$ opens the door onto a new area. We start with the following:

Definition: Let $X$ be a Banach space, let $E$ be a subspace of $X$ and let $\lambda \geq 1$. We say that the pair $(E, X)$ has the $\lambda-\mathbf{C}(\mathbf{K})$ Extension Property $(\lambda-\mathrm{C}(\mathrm{K})$ EP, for short) if for every compact Hausdorff space $\mathrm{K}$, any operator $\mathrm{T}: \mathrm{E} \rightarrow \mathrm{C}(\mathrm{K})$ admits an extension $\hat{\mathrm{T}}: \mathrm{X} \rightarrow \mathrm{C}(\mathrm{K})$ with $\|\hat{\mathrm{T}}\| \leq$ $\lambda\|\mathrm{T}\|$. The pair $(\mathrm{E}, \mathrm{X})$ has the $\mathbf{C}(\mathbf{K}) \mathbf{E} \mathbf{P}$ if it has the $\lambda-\mathrm{C}(\mathrm{K}) \mathrm{EP}$ for some $\lambda \geq 1$.

The restriction of the range space to the family of $\mathrm{C}(\mathrm{K})$ spaces provides us with a simple but effective tool in the form of the following.

\section{References}

1. Lindenstrauss, J. and Petczyński, A., "Contributions to the theory of the classical Banach spaces”, J. Funct. Anal. 8 pp. 225-249, (1971)

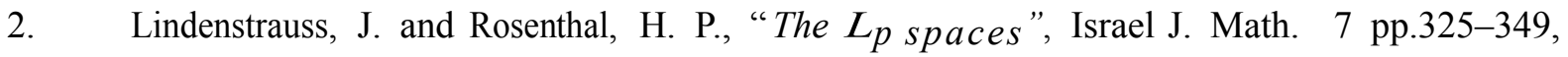
(1969)

3. Zippin, M., “The Separable extension problem”, Israel J. Math. 26 pp. 372-387, (1977)

4. Zippin, M., "The finite dimensional P $\lambda$ space for small $\lambda$ ”, Israel J. Math. pp.359-364, (1981)

5. Zippin, M., "Correction to the finite dimensional $P \lambda$ spaces with small $\lambda$ ”, Israel J. Math.48 pp. 255-256, (1984)

6 Zippin, M., “Applications of E. Michael's continuous selection theorem to operator extension problems", Proc. AMS 127 (1999).

7. Zippin, M., “On some subspaces of Banach spaces whose duals are $L_{1}$ spaces”, Proc. AMS 23 pp. 378-385, (1969) 